\title{
Redes Sociales de Video: análisis y comparación de la navegación
}

\section{Social video networks: analysis and comparison of navigation}

\author{
Cinthia Lima, Sandra Casas, Graciela Vidal \\ cinty.calderon.15@gmail.com,scasas@unpa.edu.ar,vidalgracielaunpa@gmail.com \\ GISP - Instituto de Tecnología Aplicada, Universidad de la Patagonia Austral
}

Recibido: 11/02/2019. Aceptado: 05/07/2019

\begin{abstract}
RESUMEN
En la última década los sitios web cuyo principal contenido son los videos, se han constituido como redes sociales de video (RSV). La popularidad de estas plataformas radica en el hecho de que millones de personas diariamente navegan por sus páginas, buscando, reproduciendo, subiendo, comentando y compartiendo videos propios y/o de otros usuarios. La navegación o sistemas de navegación de un sitio es uno de los factores que permite a los usuarios comprender y usar el sitio, pero además refleja una estructura organizativa del mismo. En este trabajo se presenta el análisis y comparación de la navegación de las RSV Youtube, Vimeo, Dailymotion, Metacafe, Flooxer y Break. El estudio propone dos dimensiones de análisis. Por un lado, la estructura de niveles y posibilidades de navegación horizontal y vertical para la cual se utiliza como herramienta de representación de las RSV los mapas de sitio (sitemaps), y por el otro una comprobación de guías de diseño y recomendaciones de navegabilidad, para lo cual se elaboró una lista de verificación específica. Este informe aporta tanto información cualitativa como cuantitativa, que identifica debilidades y fortalezas de las RSV seleccionadas.
\end{abstract}

Palabras clave: redes sociales de video; navegación; web.

\begin{abstract}
In the last decade websites whose main content are videos, have been constituted as social video networks (SVNs). The popularity of these platforms lies in the fact that millions of people daily browse their pages, searching, reproducing, uploading, commenting and sharing their own or other users' videos. The navigation or navigation systems of a site is one of the factors that allows users to understand and use the site, but also reflects the organizational structure of it. This paper presents the analysis and comparison of the navigation of the SVNs YouTube, Vimeo, Dailymotion, Metacafe, Flooxer and Break. The study proposes two dimensions of analysis. On one hand, the structure of levels and possibilities of horizontal and vertical navigation for which site maps (sitemaps) are used as a tool for representing SVNs, and on the other a check of design guidelines and navigability recommendations, for which a specific checklist was developed. This report provides both qualitative and quantitative information, which identifies weaknesses and strengths of the selected SVNs.
\end{abstract}

Key words: video social network; navigation; web. 


\section{INTRODUCCIÓN}

En la actualidad visitar sitios web es algo de todos los días. Un sitio web está formado por un conjunto de páginas vinculadas que comparten un único nombre de dominio, que es el nombre único que identifica a un sitio en Internet. Toda página web está compuesta por múltiples elementos como lo son textos, imágenes, audios, videos y otros contenidos multimedia, en la mayoría de los casos se navega dentro de la página o sitio web mediante hipervínculos ${ }^{1}$ que contienen los elementos de la misma. Existen diversos sitios web de video, pero los que han tomado mayor relevancia recientemente son los sitios de redes sociales. Dentro de estos sitios se encuentra YouTube, Facebook, Twitter, entre otros. Estas redes sociales se caracterizan por que sus usuarios constantemente comparten todo tipo de contenidos multimedia ya sean fotos, videos, texto, audio, etc.

Particularmente las redes sociales de video (RSV) han adquirido una gran popularidad, la cual ha crecido exponencialmente en los últimos años. Estas redes sociales tienen como característica que el principal tipo de contenido multimedia que se comparte son videos. Algunas de ellas son el ya conocido YouTube, nombrado anteriormente, Vimeo y Dailymotion. En la mayoría de ellos los usuarios pueden subir videos y compartirlos con el resto de la comunidad. Las comunidades de estos sitios suelen ser muy grandes, recientemente un estudio de We are social ${ }^{2}$ junto con Hootsuite mostró que Youtube tiene 1,900 millones de usuarios (octubre 2018), ocupando el segundo lugar en una escala junto con plataformas sociales. Esto demuestra el nivel de popularidad y uso de este tipo de sitio web hasta la fecha. Uno de los aspectos fundamentales para la buena organización y visualización de la información de los sitios web son los sistemas de navegación, que se presentan como conjuntos de enlaces cuya finalidad consiste tanto en permitir la navegación por los contenidos, como presentar al usuario la estructura de la información para facilitar la comprensión y uso del sitio. Así la navegación forma parte de una estructura más compleja conocida como la arquitectura de información web (Morrogh, E. 2002) y tiene considerable influencia en la usabilidad del sitio (Nielsen, J. 2000). Si bien se hicieron esfuerzos en el área de investigación mediante el análisis de la organización y visualizaciones de la información en sitios web (Herrera, R. y Codina L. 2015; Hofmeister C. y Han M. 2006), no se ha realizado algún estudio sobre las redes sociales de video en específico.

Una herramienta o recurso usado para representar la estructura de los sitios web e incluso mejorar la navegación de los mismos son los mapas de sitio (sitemaps). Los sitemaps pueden proveer una gran visualización ofreciendo un suplemento útil para las funciones de navegación principales en un sitio web (Nielsen J. 2008).

El objetivo de este trabajo consiste en explorar sitios web de videos o redes sociales de video, con el fin de obtener información sobre la navegabilidad de estos sitios. El trabajo se realiza sobre seis RSV, mediante el análisis de la estructura a través de la navegabilidad. Para ello se utiliza como herramienta los sitemaps, y mediante su construcción y procesamiento se obtiene información comparativa y se completa con la aplicación de una lista de verificación de navegabilidad aplicada a las RSV elegidas.

La estructura del artículo se organiza de la siguiente forma. En la Sección 2 se dará una definición general de navegación. En la Sección 3 se presentan los conceptos relacionados a sitemaps. La Sección 4 muestra los materiales y métodos utilizados para el trabajo. En la sección 5 se muestran los resultados obtenidos en el trabajo. Finalmente, se presentan las conclusiones y el trabajo futuro en la sección 6.

\footnotetext{
${ }^{1}$ Enlace asociado a un elemento de una página, al cual luego de hacerle click redirige hacia otra página.

${ }^{2}$ https://wearesocial.com/blog/2018/10/the-state-of-the-internet-in-q4-2018
} 


\section{NAVEGACIÓN}

La "falta de navegabilidad" ha sido señalada como la causa número uno de insatisfacción del usuario (Fleming, J. 1998; Nielsen, J. 1999), y como un factor que incide fuertemente en la calidad de los sitios web. El sistema de navegación o simplemente la navegación de un sitio web permiten a los visitantes moverse de una forma cómoda por las diferentes secciones y páginas que componen el sitio. Mediante la utilización de ciertos recursos de visualización, ofrecen un método de orientación para que los usuarios puedan acceder a los contenidos del sitio web sabiendo en cada momento dónde se encuentran, de dónde vienen y hacia dónde pueden ir dentro de la estructura de esa web.

Los principales tipos de sistemas de navegación web son: sistemas constantes, locales y contextuales. Los sistemas constantes (o globales) de navegación suelen encontrarse incorporados en la mayoría de páginas, en forma de una barra de navegación horizontal en la parte superior. Permiten que el usuario se oriente y sepa en todo momento dónde se encuentra y dónde puede ir. Acostumbran a permitir el acceso a las páginas o contenidos clave o más importantes del sitio web haciendo clic en partes de la barra. No suelen reflejar la jerarquía estructural entre los contenidos concretos de una página, aunque sí ofrecen una idea de cómo se encuentra estructurado el sitio web completo. Como este tipo de sistemas tiene consecuencias directas sobre la usabilidad del sitio en el que se implantan, acostumbran a ser sometidos a numerosos test y puestos a prueba antes de incorporarlos definitivamente.

Los sistemas locales de navegación son recursos compuestos normalmente por una barra vertical (aunque en algunas ocasiones se diseña con forma horizontal) de navegación que complementan los anteriores sistemas constantes y sirven para explorar una página concreta. También orientan al usuario para que sepa qué hay cerca de la página visitada y dónde puede ir.

Existen ciertas relaciones entre contenidos que se escapan o que no pueden ser recogidas por los sistemas constantes y locales descritos. Por ello, los sistemas contextuales de navegación permiten relacionar un contenido que se está visitando con otro contenido en otra página mediante un enlace o hipervínculo ligado a un literal o icono.

Existen diversas guías y directrices para el diseño del sistema de navegación de un sitio web (Kingsburg, J.R. y Andre, A.D., 2004; Hornof, A.J. y Halverson, T., 2003; Nielsen, J. y Tahir, M., 2002; Badre, A.N., 2002; Nielsen, J., 2011; Hull, S.S., 2004) en particular en este trabajo se utiliza una selección proporcionada por Leavitt M.O. y Shneiderman, B. (2004).

\section{SITEMAPS}

Los sitemaps ofrecen una visión global del sitio web, de su contenido y estructura general. Un sitemap es un archivo con una lista de las páginas de un sitio web organizadas de manera jerárquica que puede ser accedido por buscadores o usuarios. Sirven para mejorar la manera de navegar en la página por parte de los usuarios, y por otro lado facilita una enumeración de las páginas que componen el sitio web y que los motores de búsqueda rastrean. (Sitemaps.org 2008)

La funcionalidad más antigua de los sitemaps era ofrecer una representación visual del espacio de la información con el objetivo de ayudar a los usuarios a entender a donde podían ir, es decir, como podían navegar por el sitio. Los sitemaps pueden proveer una gran visualización ofreciendo un suplemento útil para las funciones de navegación principales en un sitio web (Nielsen J. 2008). Uno de los objetivos principales que motivaban la inclusión de estos mapas en los sitios web era que facilitaban la tarea de los robots de los buscadores, mejorando la indización del sitio y por ende su posicionamiento en los resultados de 
búsqueda. Sin embargo, desde que Google introdujo el concepto de sitemap web en XML los sitemaps web tradicionales han perdido popularidad entre las tácticas de optimización para buscadores. (Montero, Y. H. y Ortega Santamaría, S. 2009)

Dentro de las utilidades que se le pueden dar a los sitemaps existen varias posibilidades en cuanto a actores y a la vez definen tipos de sitemaps, como se muestra en la Tabla 1 (Garenne, B. 2018; Montero, Y. H y Ortega Santamaría, S. 2009):

Tabla 1: Utilidad de sitemaps

\begin{tabular}{|c|c|c|}
\hline $\begin{array}{c}\text { Actor } \\
\text { involucrado }\end{array}$ & $\begin{array}{c}\text { Tipo de } \\
\text { sitemaps }\end{array}$ & Descripción de relación \\
\hline $\begin{array}{l}\text { Usuario } \\
\text { común }\end{array}$ & $\begin{array}{l}\text { Sitemap } \\
\text { HTML }\end{array}$ & $\begin{array}{l}\text { El sitemap se representa como una página HTML dentro del sitio } \\
\text { donde el usuario está navegando. En la página se encuentran } \\
\text { listadas todas las páginas web del sitio con hiperenlaces a ellas. } \\
\text { Brindan una vista de todo el contenido disponible del sitio en una } \\
\text { sola página y acceso instantáneo a ese contenido. Esto les permite a } \\
\text { los usuarios rastrear la página web del sitio que sea de su interés. } \\
\text { Estos mapas actúan como guías intangibles que ayudan al usuario } \\
\text { común a navegar por un sitio. }\end{array}$ \\
\hline $\begin{array}{l}\text { Motor de } \\
\text { búsqueda }\end{array}$ & $\begin{array}{l}\text { Sitemap } \\
\text { XML }\end{array}$ & $\begin{array}{l}\text { El sitemap se representa como un archivo XML que contiene todas } \\
\text { las URL de un sitio web, junto con metadatos adicionales como, por } \\
\text { ejemplo: última actualización, frecuencia de modificación, } \\
\text { importancia, etc. La construcción del archivo XML debe seguir } \\
\text { una serie de pautas especificadas en el protocolo de sitemaps } \\
\text { XML }{ }^{3} \text {. } \\
\text { Los motores de búsqueda (Google, Yahoo, etc) buscan archivos } \\
\text { almacenados en servidores web de acuerdo a una palabra clave de } \\
\text { búsqueda. } \\
\text { Las páginas de un sitio son indexadas en los motores de búsqueda } \\
\text { para que sean fácilmente accesibles por los usuarios cuando } \\
\text { realizan una búsqueda desde el motor. }\end{array}$ \\
\hline Webmaster & $\begin{array}{c}\text { Sitemap } \\
\text { Visual }\end{array}$ & $\begin{array}{l}\text { El sitemap se representa mediante gráficas en forma de árboles, } \\
\text { diagramas jerárquicos, carpetas con jerarquía u otro. Organizan el } \\
\text { contenido que incluye y/o va a incluir un sitio web, de manera } \\
\text { estrictamente visual. Estos sitemaps son utilizados como } \\
\text { herramienta de planificando del proyecto, de administración cuando } \\
\text { se está construyendo el sitio web y de mantenimiento luego de } \\
\text { haber terminado el sitio. Los sitemaps visuales permiten tener una } \\
\text { idea general y exclusivamente visual acerca del objetivo del sitio } \\
\text { web. } \\
\text { Utilizados por webmaster y diseñadores. }\end{array}$ \\
\hline
\end{tabular}

Durand y Kahn, 1998 citados por Pilgrim, C. J (2007) clasificaron diversas estructuras visuales que pueden ser usadas para crear sitemaps web como estructuras basadas en gráficos, que son descritas como hipertexto puro y son generalmente implementadas como diagramas nodo-relación; estructuras jerárquicas, este tipo restringe a los basados en gráficos, mostrando claramente las relaciones superiores/inferiores; y estructuras espaciales, usan un sistema de coordinado explicito donde los puntos de navegación son posicionados en algunos ejes de acuerdo al valor relativo de algunos atributos cuantificables o características. Estos esquemas de clasificación destacan que los factores clave que deben ser considerados en una taxonomía de diseños de sitemaps son la estructura, el método de generación y el nivel de interactividad.

\footnotetext{
${ }^{3}$ https://www.sitemaps.org/es/protocol.htm
} 
De estos tres factores los que son de interés para nosotros son la estructura y el método de generación.

La estructura de los sitemaps la clasifican en:

- Representaciones red: son las que producen estructuras altamente complejas. Estos tipos de sitemaps son también típicamente muy complejos para el uso real (Nielsen, 1990). Durand and Kahn (1998) también reclaman que esta red "pura" muestra mucha información para un lector para una fácil asimilación, ellas carecen de una significativa topología, y que la posición relativa de los nodos en el mapa es muy arbitraria.

- Representación jerárquica: restringen la forma de estructura red quitando algunas conexiones a través de categorías semánticas o estructurales. La navegación es facilitada imponiendo una topología más simple sobre una estructura compleja (Parunak, 1989 citado por Pilgrim, C. J. 2007) mediante un proceso de filtrado de conexiones. La ventaja de las jerarquías es que apoyan la toma de decisiones en la navegación por la reducción del número de alternativas que deben ser considerados en cualquier momento (Norman K., 1991) y son bien conocidos por sus aplicaciones en varios dominios (Durand and Kahn, 1998). Incluso en la topología más aleatoria, los usuarios tenderán a imponer una estructura jerárquica para tratar de dar sentido a las estructuras (McNamara et al., 1989 citado por Pilgrim, C. J. 2007), sugiriendo que los usuarios son mejor soportados proveyendo una abstracción jerárquica o de la estructura del sitio o de las principales categorías de información. Las representaciones jerárquicas pueden limitar la flexibilidad (Durand y Kahn, 1998) y se podrían perder representaciones importantes de relaciones entre las secciones de la red.

- Representación categórica: presentan los sitemaps como una lista o grupo de las principales categorías de información encontrada en el sitio web. La categorización está basada generalmente en semántica en lugar que cualquier otro factor estructural.

El método de generación de los sitemaps puede ser automático o manual. Los sitemaps pueden generarse automáticamente al utilizar técnicas computacionales que escanean por completo topología de un sitio, anotando todos los nodos y enlaces. La generación manual de sitemaps se logra utilizando texto o herramientas gráficas. (Pilgrim, C. J. 2007)

\section{MATERIALES Y MÉTODOS}

En esta Sección se presentan las técnicas, herramientas y recursos empleados y aplicados en el estudio. La Tabla 2 presenta los RSV seleccionados para realizar el análisis que se propone en este proyecto y algunas características.

Tabla 2: RSV seleccionadas

\begin{tabular}{|c|c|c|c|c|}
\hline Nombre & URL & $\begin{array}{c}\text { Año de } \\
\text { creación }\end{array}$ & País de Origen & $\begin{array}{c}\text { ¿Posee } \\
\text { sitemap? }\end{array}$ \\
\hline Youtube & www.youtube.com & 2005 & Estados Unidos, California & No \\
\hline Vimeo & www.vimeo.com & 2004 & $\begin{array}{c}\text { Estados Unidos, New York } \\
\text { City }\end{array}$ & $\mathrm{Si}^{4}$ \\
\hline Dailymotion & ww.dailymotion.com & 2005 & Francia, París & No \\
\hline Metacafe & wwww.metacafe.com & 2002 & Estados Unidos, California & No \\
\hline Flooxer & www.flooxer.com & 2015 & Estados Unidos, California & No \\
\hline Break & ww.break.com & 1998 & Estados Unidos & No \\
\hline
\end{tabular}

${ }^{4}$ https://vimeo.com/site_map 


\subsection{Desarrollo y análisis de sitemaps}

Para el desarrollo de los sitemaps se optó por las representaciones, Sitemaps visuales y Sitemaps XML (Tabla 1). Los sitemaps de las RSV fueron construidos particularmente para el desarrollo de este trabajo, ya que como se indica en la Tabla 2 las RVS no tienen disponibles los sitemaps o al menos no de manera pública, o en el caso de Vimeo, el formato no se adecua a las necesidades del trabajo.

La construcción de los sitemaps de las RSV se realizó en cinco etapas:

1. Representación visual: se construyeron sitemaps visuales de manera manual. Para el desarrollo de estos primeros sitemap se optó por graficarlos en forma de árboles. La estructura que se representa es la que se accede desde un perfil de usuario registrado en la RSV, excepto la de Break, sitio en el cual no se logró acceder con usuario. Para la representación de estos sitemaps se eligió el enfoque categórico en el cual se agruparon las páginas de los sitios de acuerdo a una categoría. Los nombres y cantidad de categorías se fueron definiendo de acuerdo a la estructura que mostraba cada sitio en su página principal, y como éste definía las secciones.

Para armar la estructura de estos sitemaps se descartaron los canales, los millones de videos de los sitios, listas de reproducción, y demás componentes de los sitios de esta índole que no aportan información útil para este estudio. La Figura 1 ejemplifica esta representación. Del lado izquierdo de la figura se muestra una sección del panel izquierdo de opciones del sitio Metacafe. En el lado derecho de la Figura está ubicada la estructura que se generó cuya raíz fue denominada Home: Metacafe, y en el primer nivel del árbol, se ubican los elementos Lates, Popular, Trendings, Categories, etcétera. De "Categories" se desprenden Art Animation, Comedy, Cool Commercials, y los demás. De esta manera se realizaron todos los sitemaps visuales, se identificaban en la página los nombres de los hipervínculos y en base a eso se creaba el árbol del sitemap.

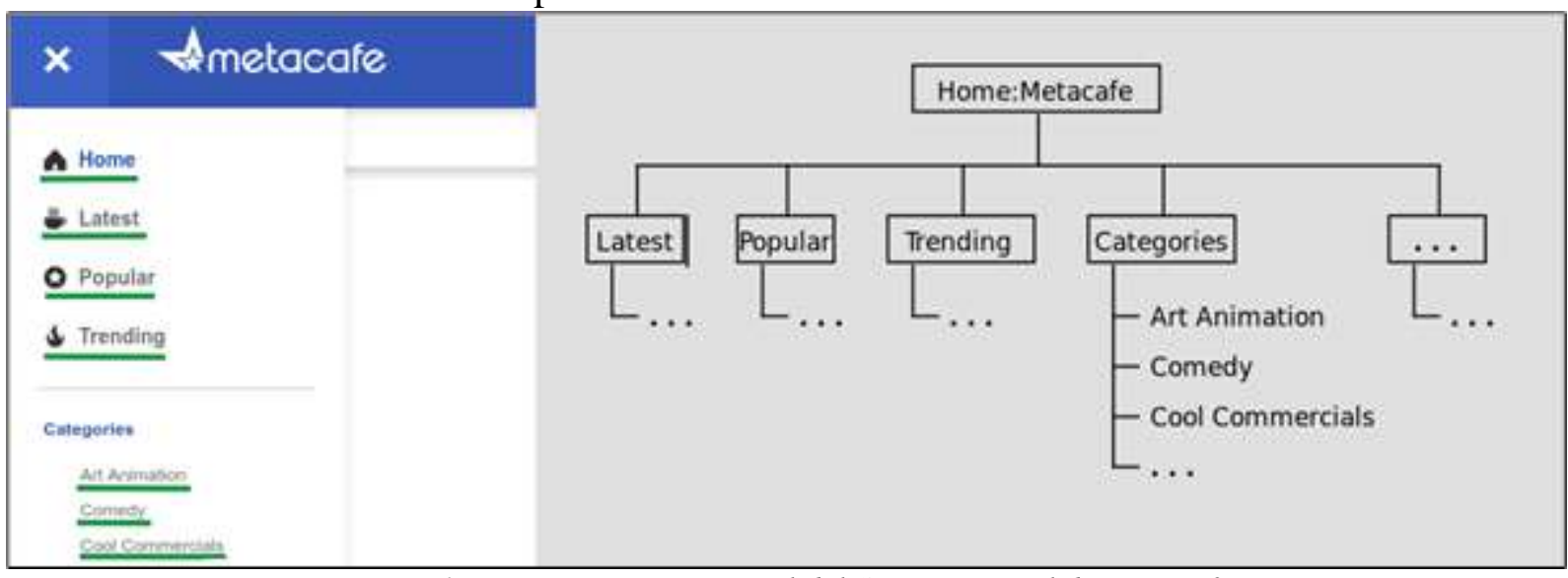

Figura 1: Representación parcial del Sitemap visual de Metacafe

2. Representación XML: se crearon sitemaps XML de las RSV, de manera automática con la ayuda de la herramienta online XML Sitemaps Generator ${ }^{5}$. La herramienta generó los sitemaps de manera automática de 5 de las RSV, ya que no pudo generar el de Flooxer. Estos sitemaps XML están basados en los RSV sin perfil de usuario registrado. Sin embargo, esto no generó inconvenientes ya que pudo rescatar buena parte de la estructura de las RSV, además de videos, canales, etc. Una vez obtenidos todos los sitemaps XML se procedió a analizar cada uno de ellos para extraer únicamente la estructura general de interés para el trabajo, lo cual implicó descartar de los XML, cientos de url de videos y canales específicos que no eran de relevancia.

Luego de realizar el descarte, cada uno de los XML de las RSV quedó compuesto por elementos del tipo "url" como se puede ver en la Figura 2. El elemento "url" contiene "loc" la

${ }^{5}$ https://www.xml-sitemaps.com/ 
dirección url, "lastmod" última fecha de modificación y "priority", es decir prioridad. Para este trabajo el único elemento de interés es "loc" es decir todas las url del sitio que puede extraer la herramienta.

<url>
$\quad<$ loc $>$ https://Ww. youtube. com/feed/history</loc $>$
$\quad<$ lastmod $>2018-06-13$ T02:19:39+00:00</lastmod $>$
$\quad<$ priority $>0.80</$ priority>
$</$ url>

Figura 2: Parte del XML generado por la herramienta

Se decidió realizar esta representación automática para asegurar que con los sitemaps manuales se estaba representando la estructura de los sitios de manera correcta. Esta verificación es en la que se trabaja en la siguiente etapa.

3. Contrastación de esquemas: se procedió a contrastar los sitemaps generados en las dos etapas anteriores. Se analizaron y compararon los sitemaps visuales y los sitemaps XML ya que ambos brindaban la estructura de los RSV estudiados. Ambos sitemaps presentaron un conjunto de elementos en común. El objetivo en esta tercera etapa fue generar un sitemap XML con un enfoque categórico igual que el Sitemap visual, pero con una estructura de RSV con perfil registrado. Estos sitemaps no fueron generados de manera manual, ni de manera automática, sino de una manera mixta trabajado con los sitemaps generados en la etapa 1 y 2 . Para esta tercera etapa es importante explicar la estructura que se eligió para el nuevo archivo XML creado:

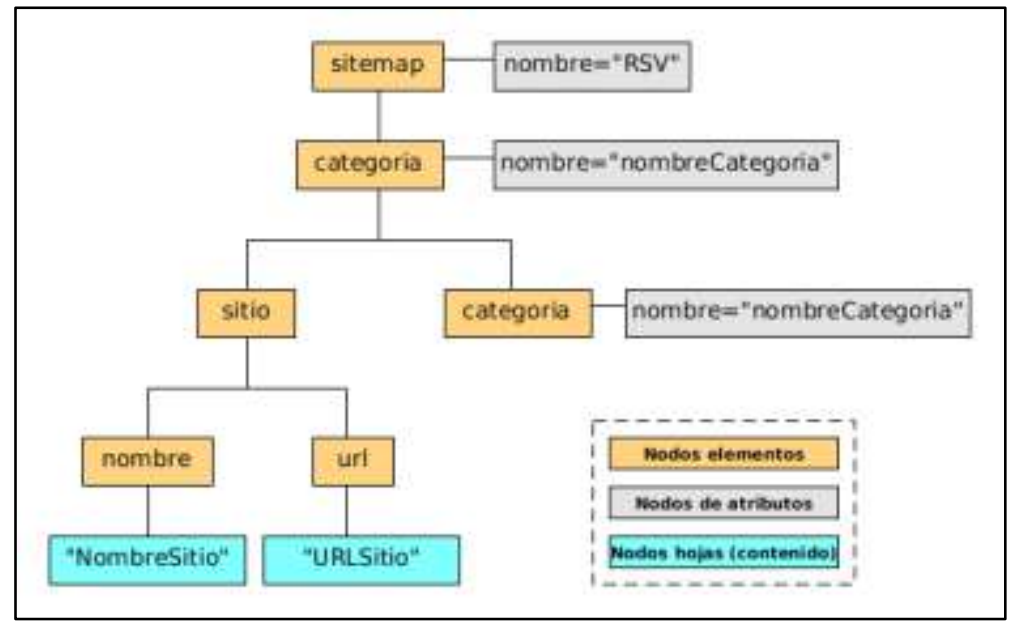

Figura 3: Estructura y elementos del XML

Como se puede ver en la Figura 3, el archivo XML consta de 5 tipos de elementos, el primero es sitemap que es el elemento raíz y tiene un atributo nombre donde se debe especificar el nombre de la RSV. Luego se encuentra el elemento categoría, porque como ya se mencionó anteriormente se eligió el enfoque categórico para representar la estructura de los sitios, este elemento también posee un atributo nombre en la cual se debe especificar el nombre de la misma. A partir de aquí el elemento categoría puede tener un elemento sitio y/o un elemento categoría. En el caso de tener un elemento categoría nuevamente, este puede tener un elemento sitio y/o categoría nuevamente y así sucesivamente (recursivo). En cuanto al elemento sitio está compuesto por otros dos elementos los cuales son nombre y url, estos ya contienen información (contenido, no atributos), tienen nodos hojas de contenido "NombredeSitio" y "URLSitio".

Para esta contrastación de esquemas, uno visual y otro XML, se identificó en primera instancia que nombres definidos en el sitemap visual se correspondían con las url especificadas en el XML generado por la herramienta. Esto quiere decir, identificar que nombre de hipervínculos definidos en el sitemap visual tenía su dirección de url en el sitemap 
XML generado por la herramienta, por ejemplo, como se ve en la Figura 4, hipervA con urlA, hipervB con url-B y hipervC con url-C.

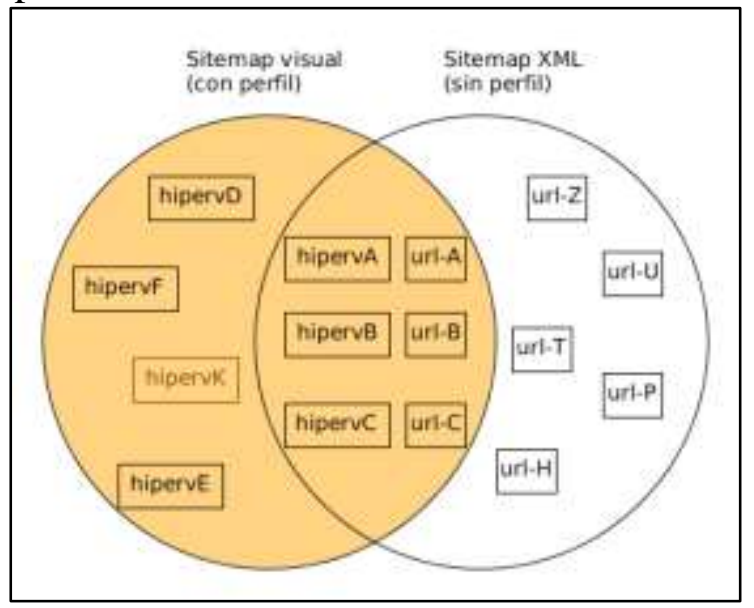

Figura 4: Elementos comunes entre sitemaps

Como el objetivo era generar un nuevo sitemap XML basado en una estructura con perfil a partir de los dos generados anteriormente, este nuevo XML contiene todos los elementos comunes entre ambos sitemaps, más los identificados en el sitemap visual con perfil que no pudo detectar la herramienta. Se encontraron múltiples elementos que cumplían con la condición de correspondencia.

4. Unión de esquemas y esquema final: después de obtener los XML para cada una de las RSV, se generó un único XML que se compone de todos los sitemaps XML. Se realizó esta unión para facilitar y agilizar los procesamientos posteriores. Este último XML estaba delimitados por la etiqueta sitemaps y dentro de ella se encontraban los siete sitemaps generados en la etapa tres.

5. Análisis de los sitemaps XML: se utilizó la herramienta XQuery ${ }^{6}$, que permite hacer consultas sobre archivos XML para obtener información acerca de estas RSV. Las consultas se hicieron sobre una herramienta online ${ }^{7}$ que permite ejecutar consultas XQuery. Con las consultas se obtuvo dos tipos de datos que son la anchura y la profundidad de cada uno de los sitemaps XML.

Las consultas realizadas fueron con el fin de obtener esta información acerca de la navegabilidad en cada RSV.

\subsection{Navegabilidad de los sitios de video}

Además de desarrollar los sitemaps XML, para analizar la navegabilidad de los sitios web, también se elaboró una "lista de verificación" de navegabilidad. La lista de verificación fue creada en base a una serie de guías propuestas por Leavitt M. O. y Shneiderman, B. (2004) las cuales son ponderadas por dos indicadores: uno que indica el nivel relativo de importancia (NRI) y otro que indica el nivel de fuerza de evidencia (NFE), ambos están en un rango de 1 a 5. A continuación se detallarán todos los elementos de la lista de verificación con sus respectivos indicadores.

G01: No dirigir a los usuarios a páginas que no tienen opciones de navegación (NRI: 4, NFE: 2)

Los diseñadores no deben crear páginas web que deshabiliten el botón Atrás del navegador. Desactivar el botón Atrás puede provocar confusión y frustración para los usuarios, e inhibe drásticamente su navegación. Y si dichas páginas se incorporan a un sitio web, deben tener

\footnotetext{
${ }^{6}$ https://www.w3.org/XML/Query/

7 http://www.xpathtester.com/xpath
} 
algún mecanismo o botón de control que cierre la ventana o pestaña y devuelva al usuario a la ventana del navegador original.

G02: Diferenciar claramente los elementos de navegación entre sí, en forma agrupada y colocados en un lugar coherente y fácil de encontrar en cada página (NRI: 4, NFE: 3)

Se debe utilizar un esquema de navegación común en todo el sitio web para ayudar a los usuarios a aprender y comprender la estructura del sitio web. Se debe utilizar el mismo esquema de navegación en todas las páginas al ubicar de forma consistente las pestañas, los encabezados, las listas, la búsqueda, el mapa del sitio, etc. Se deben ubicar los elementos críticos de navegación en los lugares que sugieran accesibilidad.

Los elementos de navegación deben ser lo suficientemente diferentes entre sí para que los usuarios puedan comprender la diferencia entre su significado y destino. La agrupación reduce la cantidad de tiempo que los usuarios necesitan para localizar e identificar los elementos de navegación.

Deben colocarse las opciones relevantes juntas y también utilizar listas verticales.

G03: En páginas largas, proporcionar una "lista de contenidos" con enlaces que lleven a los usuarios al contenido correspondiente más abajo en la página (NRI: 4, NFE: 3)

Para páginas largas con varias secciones distintas que no están visibles desde la primera pantalla, se debe agregar una lista corta y seleccionable de las secciones (enlaces de anclaje) en la parte superior de la página. Los "enlaces de anclaje" pueden servir para dos propósitos: proporcionan un esquema de la página para que los usuarios puedan determinar rápidamente si contiene la información deseada y permiten a los usuarios navegar rápidamente a información específica.

Dado que los 'enlaces de anclaje' permiten un enlace directo al contenido debajo de la primera pantalla, también son útiles para que los usuarios obtengan información específica rápidamente cuando llegan de una página completamente diferente.

G04: Proporcionar indicadores para que los usuarios sepan dónde están en el sitio web (NRI: 4, NFE: 2)

El feedback proporciona a los usuarios la información que necesitan para comprender dónde se encuentran dentro del sitio web y para pasar a la siguiente actividad. Los ejemplos de feedack incluyen proporcionar información sobre la ruta y la jerarquía (es decir, "breadcrumb"), hacer coincidir el texto del enlace con el encabezado de la página de destino y crear direcciones URL relacionadas con la ubicación del usuario en el sitio. Otras formas de feedback incluyen cambiar el color de un enlace que ya se ha visitado y usar otras indicaciones visuales para indicar la parte activa de la pantalla.

G05: Colocar los menús de navegación primarios en el panel izquierdo y los menús secundario y terciario juntos (NRI: 4, NFE: 3 )

Según estudios, los usuarios prefieren el menú principal en el panel izquierdo, y agrupar los menús secundarios y terciarios juntos, o agrupar los tres niveles del menú juntos. El mejor rendimiento y preferencia se logró cuando los tres menús se colocaron en el panel izquierdo.

G06: Las etiquetas de la pestaña son claramente descriptivas de su función o destino (NRI: 3, NFE: 3)

Los usuarios prefieren las pestañas que tienen etiquetas que son lo suficientemente descriptivas como para permitir selecciones sin errores. Se recomienda no usar etiquetas de pestañas cuando no se pueden aclarar debido a la falta de espacio.

G07: Las pestañas de navegación están ubicadas en la parte superior de la página y se parecen a versiones seleccionables de pestañas del mundo real (NRI: 3, NFE: 3)

Los usuarios pueden confundirse con el uso de las pestañas cuando no parecen pestañas del mundo real. Las pestañas del mundo real son aquellas que se parecen a las que se encuentran en un cajón de archivos. Un estudio mostró que es más probable que los usuarios encuentren y hagan clic de forma adecuada en las pestañas que parecen pestañas del mundo real. 
G08: No es necesario que los usuarios se desplacen en las páginas puramente de navegación (todo en una pantalla) (NRI: 2 , NFE: 4)

Idealmente, las páginas de solo navegación no deben contener más de una pantalla llena de información. Los usuarios no deben desplazarse por la página, incluso una pequeña distancia. Un estudio mostró que los usuarios consideraron la parte inferior de una pantalla como el final de una página, y no se desplazaron más para encontrar opciones de navegación adicionales G09: Usar los menús secuenciales para las tareas simples que se mueven hacia adelante, y usa los menús simultáneos para las tareas que de otro modo requerirían numerosos usos del botón Atrás (NRI: 2, NFE: 4)

La mayoría de los sitios web utilizan menús secuenciales que requieren que los elementos se seleccionen de una serie de menús en un orden predeterminado. Después de hacer cada selección, se abre otro menú. La elección final está limitada por la suma total de todas las elecciones anteriores.

Los menús simultáneos muestran opciones de múltiples niveles en la jerarquía de menús, lo que brinda a los usuarios la capacidad de elegir opciones del menú en cualquier orden. Los menús simultáneos a menudo se presentan en marcos, y se emplean mejor en situaciones en las que los usuarios tendrían que hacer un uso extensivo del botón Atrás si se les presenta un menú secuencial.

G10: Utilizar los sitemaps para los sitios web que tienen muchas páginas (NRI: 2, NFE: 4)

Algunos estudios sugieren que los sitemaps no necesariamente mejoran las representaciones mentales de los usuarios de un sitio web. Además, un estudio informó que si un mapa del sitio no refleja la estructura conceptual de los usuarios (o del dominio), la utilidad del mapa se reduce, por ende, si utiliza sitemaps deben estar bien representados.

G11: Proporcionar "glosses" para ayudar a los usuarios a seleccionar los enlaces correctos (NRI: 1, NFE: 2)

Los "Glosses" son frases cortas de información que aparecen de forma emergente cuando un usuario coloca el puntero de su mouse cerca de un enlace. Proporciona una vista previa de la información detrás de un enlace. Los usuarios prefieren que la información de vista previa se ubique cerca del enlace, pero que no se coloque de manera que perturbe el texto primario. Sin embargo, los diseñadores no deben confiar en los "glosses" para compensar los enlaces mal etiquetados.

G12: Proporcionar breadcrumb de manera efectiva (NRI: 1, NFE: 3)

Un estudio encontró que los participantes de la prueba que recibieron instrucción sobre el uso de breadcrumb completaron las tareas mucho más rápido que los que no lo hicieron. Este ahorro de tiempo podría resultar en una mayor productividad para los usuarios que realizan búsquedas en sitios web a diario.

De todas estas guías, G06 y G07 no son aplicables al estudio de las RSV, porque las RSV no poseen elementos en las páginas que presenten menús en forma de pestañas.

\section{RESULTADOS}

Se realizaron dos tipos de consultas, una para obtener la cantidad de niveles máximos de cada sitemap, y la cantidad total de elementos en cada nivel, y otra para conocer la anchura máxima en cada nivel. La especificación de las consultas realizadas se encuentra detallada en el Anexo.

a) Cantidad de elementos en cada nivel y profundidad: esta consulta permite conocer la cantidad de elementos, y al mismo tiempo conocer el nivel de profundidad máximo de cada RSV. Y también conocer la cantidad de elementos ya sean categorías o sitios de cada uno de los niveles. 
En la Tabla 3 se pueden ver los resultados de aplicar consultas con XQuery sobre cada uno de los sitemaps XML de las RSV. Se obtuvo que Youtube y Vimeo tienen un nivel máximo de 4, Dailymotion y Flooxer uno de 2, Metacafe uno de 3, y Break un nivel máximo de 5.

Tabla 3: Distribución de elementos

\begin{tabular}{|c|c|c|c|c|c|c|}
\hline \multicolumn{7}{|c|}{ Distribución de elementos en los sitios web } \\
\hline Nivel & Youtube & Vimeo & Dailymotion & Metacafe & Break & Flooxer \\
\hline 1 & 12 & 12 & 9 & 8 & 7 & 15 \\
\hline 2 & 37 & 61 & 33 & 39 & 39 & 70 \\
\hline 3 & 12 & 48 & 0 & 51 & 29 & 0 \\
\hline 4 & 18 & 79 & 0 & 0 & 92 & 0 \\
\hline 5 & 0 & 0 & 0 & 0 & 5 & 0 \\
\hline
\end{tabular}

b) Distribución de elementos en cada nivel

Además de conocer la cantidad de elementos de cada nivel, es necesario conocer si son páginas, es decir nodos terminales, o son categorías que incluyen sitios, es decir, nodos no terminales. Es decir, no se conoce la distribución de los elementos dentro de cada nivel. A continuación, se hicieron consultas específicas sobre cada RSV para conocer la estructura de cada sitemap XML. Con estas consultas se buscó conocer la anchura máxima de cada nivel en las RSV.

Para la mejor comprensión de los resultados obtenidos se va a explicar de manera detallada, acompañada con un gráfico, los resultados obtenidos del sitemap XML de Youtube. Mientras que para las otras RSV se especificará únicamente los resultados.

Youtube

Esta RSV presenta en el primer nivel 12 nodos no terminales (categorías), cada una de esas categorías al ser nodos no terminales contiene más nodos, algunos terminales (sitios) y otros no terminales (categorías). Esto se repite cada vez que se presenta una categoría. En el nivel 2 se pueden ver diferentes valores, cada uno de ellos se corresponde con cada uno de los 12 nodos del nivel 1, es decir, el primer nodo del nivel 1 tiene 1 hijo, el segundo tiene 7 , el tercero tiene 2, el cuarto tiene 4 y así sucesivamente, que en total suman 37 nodos. La anchura máxima en este segundo nivel es de 7 . En el tercer nivel se presentan los hijos de 5 nodos del nivel anterior, ya que como se dijo en el nivel 2 puede haber nodos terminales y no terminales, por ende 5 nodos de los 37 del nivel 2 eran no terminales y sus hijos son 5 para el primero, 1 para el segundo, etc. La anchura máxima para este nivel es de 5. En el cuarto nivel se tienen 18 nodos terminales que pertenecen a un solo nodo no terminal del nivel 3 , esto significa que de los 12 nodos del nivel 3, 1 era no terminal y el resto terminales (Tabla 4 inciso a- y Figura 1). La anchura máxima es de 18 y la mínima es de 1.

Tabla 4: Distribución de elementos por niveles en cada RSV

\begin{tabular}{|c|c|c|c|c|c|c|c|c|c|c|c|c|c|c|c|c|c|}
\hline Nivel & \multicolumn{16}{|c|}{ RSV } & Total \\
\hline \multicolumn{18}{|c|}{ a) Youtube } \\
\hline N1 & 12 & & & & & & & & & & & & & & & & 12 \\
\hline N2 & 1 & 7 & 2 & 4 & 2 & 4 & 1 & 6 & 7 & 1 & 1 & 1 & & & & & 37 \\
\hline N3 & 5 & 1 & 1 & 2 & 3 & & & & & & & & & & & & 12 \\
\hline $\mathrm{N} 4$ & 18 & & & & & & & & & & & & & & & & 18 \\
\hline \multicolumn{18}{|c|}{ b) Vimeo } \\
\hline N1 & 12 & & & & & & & & & & & & & & & & 12 \\
\hline $\mathrm{N} 2$ & 1 & 1 & 1 & 11 & 17 & 6 & 2 & 7 & 2 & 6 & 3 & 4 & & & & & 61 \\
\hline N3 & 3 & 3 & 3 & 3 & 3 & 3 & 3 & 3 & 3 & 3 & 3 & 3 & 3 & 3 & 3 & 3 & 48 \\
\hline N4 & 6 & 7 & 4 & 2 & 5 & 3 & 3 & 3 & 8 & 3 & 8 & 2 & 7 & 7 & 3 & 8 & 79 \\
\hline
\end{tabular}




\begin{tabular}{|c|c|c|c|c|c|c|c|c|c|c|c|c|c|c|c|c|c|c|}
\hline Nivel & \multicolumn{17}{|c|}{ RSV } & Total \\
\hline \multicolumn{19}{|c|}{ c) Dailymotion } \\
\hline N1 & 9 & & & & & & & & & & & & & & & & & 9 \\
\hline N2 & 1 & 7 & 1 & 1 & 3 & 6 & 5 & 4 & 5 & & & & & & & & & 33 \\
\hline \multicolumn{19}{|c|}{ d) Metacafe } \\
\hline N1 & 8 & & & & & & & & & & & & & & & & & 8 \\
\hline N2 & 1 & 2 & 2 & 2 & 7 & 17 & 5 & 3 & & & & & & & & & & 39 \\
\hline N3 & 3 & 3 & 3 & 3 & 3 & 3 & 3 & 3 & 3 & 3 & 3 & 3 & 3 & 3 & 3 & 3 & 3 & 51 \\
\hline \multicolumn{19}{|c|}{ e) Break } \\
\hline N1 & 7 & & & & & & & & & & & & & & & & & 7 \\
\hline $\mathrm{N} 2$ & 1 & 1 & 18 & 1 & 13 & 2 & 3 & & & & & & & & & & & 39 \\
\hline N3 & 2 & 1 & 2 & 1 & 2 & 2 & 2 & 2 & 1 & 1 & 1 & 2 & 2 & 2 & 2 & 2 & & 29 \\
\hline N4 & 10 & 14 & 6 & 4 & 4 & 6 & 6 & 12 & 2 & 7 & 16 & 5 & & & & & & 92 \\
\hline N5 & 5 & & & & & & & & & & & & & & & & & 5 \\
\hline \multicolumn{19}{|c|}{ f) Flooxer } \\
\hline N1 & 15 & & & & & & & & & & & & & & & & & 15 \\
\hline N2 & 1 & 1 & 1 & 1 & 1 & 1 & 1 & 1 & 1 & 1 & 52 & 1 & 5 & 1 & 1 & & & 70 \\
\hline
\end{tabular}

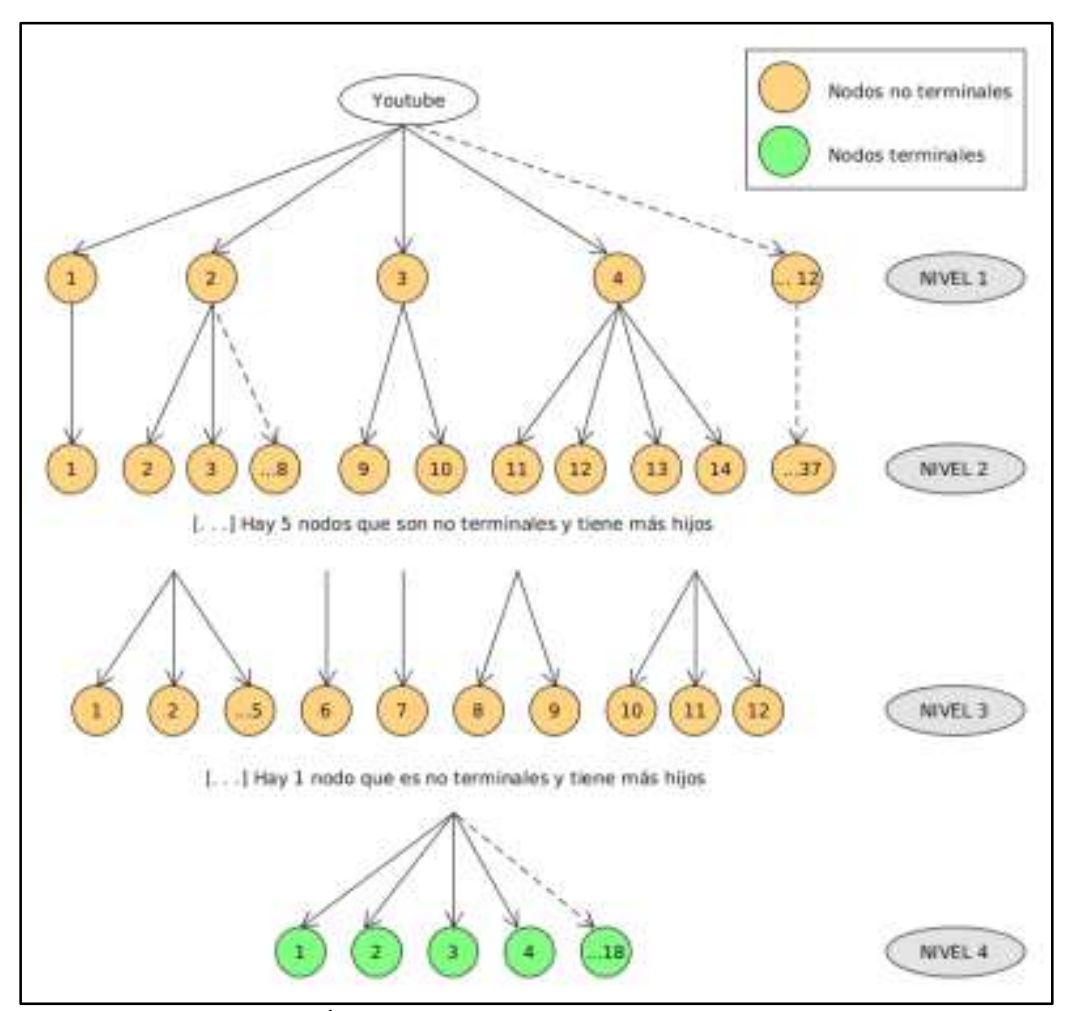

Figura 5: Árbol de niveles de Sitemap XML Youtube

En Vimeo la anchura máxima en el primer nivel es de 12, en el segundo de 17, en el tercero de 3 y en el cuarto de 8. La anchura máxima es 17 y la mínima 1. En la RSV Dailymotion la anchura máxima en el primer nivel es de 9 y en el segundo de 7. La anchura máxima es 9 y la mínima 1. En Metacafe la anchura máxima en el primer nivel es de 8, en el segundo de 17, en el tercero de 3. La anchura máxima es 17 y la mínima 1. En Break la anchura máxima en el primer nivel es de 7, en el segundo de 18, en el tercero de 2, en el cuarto de 16 y en el quinto de 5. La anchura máxima es 18 y la mínima 1. Y por último en Flooxer la anchura máxima en el primer nivel es de 15 y en el segundo de 52. La anchura máxima es 52 y la mínima 1. 


\subsection{Resultados al aplicar la guía de navegación}

En el análisis de las 10 guías de navegación se efectuó a partir de la exhaustiva exploración y observación de cada una de las RSV seleccionadas y a partir de una lista de verificación (Tabla 5).

Tabla 5: Resultados al aplicar la lista de verificación de navegabilidad

\begin{tabular}{|c|c|c|c|c|c|c|c|c|c|}
\hline $\begin{array}{c}\mathrm{G} \\
\text { uí } \\
\mathrm{a}\end{array}$ & $\begin{array}{l}\mathrm{N} \\
\mathrm{R} \\
\mathrm{I} \\
\end{array}$ & $\begin{array}{l}\mathrm{N} \\
\mathrm{F} \\
\mathrm{E}\end{array}$ & Youtube & Vimeo & Metacafe & Dailymotion & Flooxer & Break & \\
\hline $\begin{array}{c}\mathrm{G} \\
01\end{array}$ & 4 & 2 & $\begin{array}{c}\text { SI } \\
\text { No dirige a los } \\
\text { usuarios a } \\
\text { páginas que no } \\
\text { les permitan } \\
\text { volver hacia } \\
\text { atrás. }\end{array}$ & $\begin{array}{c}\text { SI } \\
\text { No dirige a } \\
\text { los usuarios a } \\
\text { páginas que } \\
\text { no les } \\
\text { permitan } \\
\text { volver hacia } \\
\text { atrás. }\end{array}$ & $\begin{array}{c}\text { SI } \\
\text { No dirige a } \\
\text { los usuarios } \\
\text { a páginas } \\
\text { que no les } \\
\text { permitan } \\
\text { volver hacia } \\
\text { atrás. }\end{array}$ & $\begin{array}{c}\text { SI } \\
\text { No dirige a } \\
\text { los usuarios } \\
\text { a páginas } \\
\text { que no les } \\
\text { permitan } \\
\text { volver hacia } \\
\text { atrás. }\end{array}$ & $\begin{array}{c}\text { SI } \\
\text { No dirige a } \\
\text { los usuarios } \\
\text { a páginas } \\
\text { que no les } \\
\text { permitan } \\
\text { volver hacia } \\
\text { atrás. }\end{array}$ & $\begin{array}{c}\text { SI } \\
\text { No dirige a } \\
\text { los usuarios a } \\
\text { páginas que } \\
\text { no les } \\
\text { permitan } \\
\text { volver hacia } \\
\text { atrás. }\end{array}$ & $\begin{array}{c}\mathrm{SI}= \\
6 \\
\mathrm{NO} \\
=0\end{array}$ \\
\hline $\begin{array}{c}\mathrm{G} \\
02\end{array}$ & 4 & 3 & $\begin{array}{c}\text { SI } \\
\text { Los elementos } \\
\text { de navegación } \\
\text { se encuentran } \\
\text { agrupados, en } \\
\text { un menú } \\
\text { superior y en } \\
\text { uno lateral. } \\
\text { Tiene } \\
\text { separadores } \\
\text { entre grupos de } \\
\text { elementos que } \\
\text { están juntos } \\
\text { por afinidad }\end{array}$ & $\begin{array}{c}\text { SI } \\
\text { Presenta un } \\
\text { menú } \\
\text { superior con } \\
\text { opciones, y } \\
\text { un footer con } \\
\text { hipervínculos } \\
.\end{array}$ & $\begin{array}{c}\text { SI } \\
\text { Presenta } \\
\text { dos paneles } \\
\text { laterales } \\
\text { que tienen } \\
\text { elementos } \\
\text { de } \\
\text { navegación } \\
\text { agrupados } \\
\text { de acuerdo } \\
\text { a un fin. } \\
\text { Además, } \\
\text { una barra } \\
\text { superior con } \\
\text { opciones y } \\
\text { un footer. }\end{array}$ & $\begin{array}{c}\text { SI } \\
\text { Los } \\
\text { elementos } \\
\text { de } \\
\text { navegación } \\
\text { de } \\
\text { encuentran } \\
\text { agrupados } \\
\text { en un menú } \\
\text { superior }\end{array}$ & $\begin{array}{c}\text { SI } \\
\text { Tiene los } \\
\text { elementos } \\
\text { de } \\
\text { navegación } \\
\text { agrupados } \\
\text { casi todos } \\
\text { en un panel } \\
\text { lateral. }\end{array}$ & $\begin{array}{c}\text { NO } \\
\text { Si bien posee } \\
\text { un panel } \\
\text { lateral con } \\
\text { algunas } \\
\text { opciones de } \\
\text { navegación, } \\
\text { tiene un menú } \\
\text { superior el } \\
\text { cual no } \\
\text { agrupa los } \\
\text { elementos de } \\
\text { navegación y } \\
\text { genera una } \\
\text { lista muy } \\
\text { grande }\end{array}$ & $\begin{array}{c}\mathrm{SI}= \\
5 \\
\mathrm{NO} \\
=1\end{array}$ \\
\hline $\begin{array}{c}\mathrm{G} \\
03\end{array}$ & 4 & 3 & $\begin{array}{c}\text { NO } \\
\text { Existen } \\
\text { páginas largas } \\
\text { y no se posee } \\
\text { un índice para } \\
\text { navegar dentro } \\
\text { de la misma. }\end{array}$ & $\begin{array}{c}\text { NO } \\
\text { Existen } \\
\text { páginas } \\
\text { largas y no se } \\
\text { posee un } \\
\text { índice para } \\
\text { navegar } \\
\text { dentro de la } \\
\text { misma. }\end{array}$ & $\begin{array}{c}\text { NO } \\
\text { Existen } \\
\text { páginas } \\
\text { largas y no } \\
\text { se posee un } \\
\text { índice para } \\
\text { navegar } \\
\text { dentro de la } \\
\text { misma. }\end{array}$ & $\begin{array}{c}\text { NO } \\
\text { Existen } \\
\text { páginas } \\
\text { largas y no } \\
\text { se posee un } \\
\text { índice para } \\
\text { navegar } \\
\text { dentro de la } \\
\text { misma. }\end{array}$ & $\begin{array}{c}\text { NO } \\
\text { Existen } \\
\text { páginas } \\
\text { largas y no } \\
\text { se posee un } \\
\text { índice para } \\
\text { navegar } \\
\text { dentro de la } \\
\text { misma. }\end{array}$ & $\begin{array}{c}\text { NO } \\
\text { Existen } \\
\text { páginas largas } \\
\text { y no se posee } \\
\text { un índice para } \\
\text { navegar } \\
\text { dentro de la } \\
\text { misma. }\end{array}$ & $\begin{array}{c}\mathrm{SI}= \\
0 \\
\mathrm{NO} \\
=6\end{array}$ \\
\hline $\begin{array}{c}\mathrm{G} \\
04\end{array}$ & 4 & 2 & $\begin{array}{l}\text { SI } \\
\text { Cuando el } \\
\text { usuario se } \\
\text { posiciona en } \\
\text { cualquier } \\
\text { página, en la } \\
\text { barra de } \\
\text { navegación se } \\
\text { resalta el } \\
\text { nombre de la } \\
\text { sección del } \\
\text { sitio en donde } \\
\text { se encuentra. }\end{array}$ & NO & $\begin{array}{c}\text { SI } \\
\text { Casi en } \\
\text { todo el sitio, } \\
\text { se remarcan } \\
\text { los } \\
\text { hipervíncul } \\
\text { os de los } \\
\text { lugares del } \\
\text { sitio en } \\
\text { donde se } \\
\text { encuentra el } \\
\text { usuario } \\
\text { actualmente }\end{array}$ & $\begin{array}{c}\text { SI } \\
\text { Se utilizan } \\
\text { mecanismos } \\
\text { visuales } \\
\text { para } \\
\text { indicarle al } \\
\text { usuario } \\
\text { donde se } \\
\text { encuentra, }\end{array}$ & NO & NO & $\begin{array}{c}\mathrm{SI}= \\
3 \\
\mathrm{NO} \\
=3\end{array}$ \\
\hline
\end{tabular}




\begin{tabular}{|c|c|c|c|c|c|c|c|c|c|}
\hline $\begin{array}{l}\mathrm{G} \\
05\end{array}$ & 4 & 3 & $\begin{array}{c}\text { SI } \\
\text { Posee el menú } \\
\text { principal con } \\
\text { las opciones } \\
\text { más } \\
\text { importantes } \\
\text { ubicadas en el } \\
\text { panel } \\
\text { izquierdo. }\end{array}$ & $\begin{array}{c}\text { NO } \\
\text { No posee } \\
\text { panel lateral. }\end{array}$ & $\begin{array}{c}\text { SI } \\
\text { Posee el } \\
\text { menú } \\
\text { principal } \\
\text { con las } \\
\text { opciones } \\
\text { más } \\
\text { importantes } \\
\text { ubicadas en } \\
\text { el panel } \\
\text { izquierdo. }\end{array}$ & $\begin{array}{c}\text { NO } \\
\text { No posee } \\
\text { panel } \\
\text { lateral. }\end{array}$ & $\begin{array}{c}\text { SI } \\
\text { Posee el } \\
\text { menú } \\
\text { principal } \\
\text { con las } \\
\text { opciones } \\
\text { más } \\
\text { importantes } \\
\text { ubicadas en } \\
\text { el panel } \\
\text { izquierdo. }\end{array}$ & $\begin{array}{c}\text { NO } \\
\text { Posee panel } \\
\text { lateral, pero } \\
\text { en él no se } \\
\text { encuentra el } \\
\text { menú } \\
\text { principal del } \\
\text { sitio. }\end{array}$ & $\begin{array}{c}\mathrm{SI}= \\
3 \\
\mathrm{NO} \\
=3\end{array}$ \\
\hline $\begin{array}{l}\mathrm{G} \\
08\end{array}$ & 2 & 4 & $\mathrm{NO}$ & NO & $\begin{array}{c}\text { SI } \\
\text { Todas sus } \\
\text { páginas de } \\
\text { navegación } \\
\text { no } \\
\text { requieren } \\
\text { desplazarse } \\
\text { hacia abajo. }\end{array}$ & NO & $\mathrm{NO}$ & NO & $\begin{array}{l}\mathrm{SI}= \\
1 \\
\mathrm{NO} \\
=5\end{array}$ \\
\hline $\begin{array}{c}\mathrm{G} \\
09\end{array}$ & 2 & 4 & $\begin{array}{c}\text { SI } \\
\text { Utiliza de } \\
\text { manera } \\
\text { apropiada los } \\
\text { menús } \\
\text { secuenciales y } \\
\text { simultáneos. }\end{array}$ & $\begin{array}{c}\text { SI } \\
\text { Utiliza de } \\
\text { manera } \\
\text { apropiada el } \\
\text { menú } \\
\text { secuencial. }\end{array}$ & $\begin{array}{c}\text { SI } \\
\text { Utiliza de } \\
\text { manera } \\
\text { apropiada } \\
\text { los menús } \\
\text { secuenciale } \\
\text { s y } \\
\text { simultáneos }\end{array}$ & $\begin{array}{c}\text { NO } \\
\text { No hace } \\
\text { uso de estos } \\
\text { menús. }\end{array}$ & $\begin{array}{c}\text { SI } \\
\text { Utiliza de } \\
\text { manera } \\
\text { apropiada el } \\
\text { menú } \\
\text { secuencial. }\end{array}$ & $\begin{array}{c}\text { SI } \\
\text { Utiliza de } \\
\text { manera } \\
\text { apropiada el } \\
\text { menú } \\
\text { secuencial. }\end{array}$ & $\begin{array}{l}\mathrm{SI}= \\
5 \\
\mathrm{NO} \\
=1\end{array}$ \\
\hline $\begin{array}{l}\mathrm{G} \\
10\end{array}$ & 2 & 4 & $\mathrm{NO}$ & $\begin{array}{c}\text { SI } \\
\text { Posee un } \\
\text { enlace en el } \\
\text { footer hacia } \\
\text { el sitemap del } \\
\text { sitio. } \\
\end{array}$ & NO & $\mathrm{NO}$ & NO & NO & $\begin{array}{l}\mathrm{SI}= \\
1 \\
\mathrm{NO} \\
=5\end{array}$ \\
\hline $\begin{array}{l}\mathrm{G} \\
11\end{array}$ & 1 & 2 & $\begin{array}{c}\text { SI } \\
\text { Utiliza } \\
\text { comentarios } \\
\text { para ayudar al } \\
\text { usuario, pero } \\
\text { son muy } \\
\text { simulares a la } \\
\text { etiqueta del } \\
\text { hipervínculo. }\end{array}$ & $\begin{array}{c}\text { SI } \\
\text { Utiliza } \\
\text { comentarios } \\
\text { para ayudar } \\
\text { al usuario y } \\
\text { aportan } \\
\text { información } \\
\text { de } \\
\text { importancia. }\end{array}$ & NO & $\begin{array}{c}\text { SI } \\
\text { Utiliza } \\
\text { comentarios } \\
\text { para ayudar } \\
\text { al usuario, } \\
\text { pero son } \\
\text { muy } \\
\text { simulares a } \\
\text { la etiqueta } \\
\text { del } \\
\text { hipervínculo }\end{array}$ & NO & NO & $\begin{array}{l}\mathrm{SI}= \\
3 \\
\mathrm{NO} \\
=3\end{array}$ \\
\hline $\begin{array}{l}\mathrm{G} \\
12\end{array}$ & 1 & 3 & NO & NO & NO & $\mathrm{NO}$ & $\mathrm{NO}$ & $\begin{array}{c}\text { SI } \\
\text { Utiliza } \\
\text { breadcrum } \\
\text { cuando se } \\
\text { navega en las } \\
\text { categorías y } \\
\text { subcategorías } \\
\text { de videos. }\end{array}$ & $\begin{array}{l}\mathrm{SI}= \\
1 \\
\mathrm{NO} \\
=5\end{array}$ \\
\hline & \multicolumn{2}{|c|}{$\begin{array}{c}\text { Resum } \\
\text { en }\end{array}$} & $\begin{array}{c}\mathrm{SI}=6 \\
\mathrm{NO}=4\end{array}$ & $\begin{array}{c}\mathrm{SI}=5 \\
\mathrm{NO}=5\end{array}$ & $\begin{array}{c}\mathrm{SI}=6 \\
\mathrm{NO}=4\end{array}$ & $\begin{array}{c}\mathrm{SI}=5 \\
\mathrm{NO}=5\end{array}$ & $\begin{array}{l}\mathrm{SI}=4 \\
\mathrm{NO}=6\end{array}$ & $\begin{array}{l}\mathrm{SI}=5 \\
\mathrm{NO}=5\end{array}$ & \\
\hline
\end{tabular}




\section{CONCLUSIONES}

Las RSV, presentan una navegación escasamente profunda, la cual varía entre 2 y 5 niveles. El nivel varía de acuerdo al diseño por el que optaron los diseñadores del sitio web, es decir, cómo decidieron agrupar los elementos de navegación, cómo ordenarlos, que tipos de menús utilizar, etc. Es por eso que en cada nivel las posibilidades de navegación en cada RSV son muy distintas unas de otras. Mientras que el segundo nivel de Break presenta 52 opciones para navegar, en el mismo nivel en las demás RSV la máxima cantidad de opciones de navegación es 18. Esto indica una desequilibrada organización y distribución de los elementos de navegación en algunos sitios en comparación con otros. Una sección fundamental en estas RSV y que está presente en cinco de las seis es la de Categorías. Esta sección agrupa las categorías y a veces subcategorías de videos, por lo general es bastante amplia. Algunos sitios la organizan de tal manera que se ve reflejada en la profundidad del sitio (niveles) y otras la presentan de tal manera que influye en la anchura máxima (opciones de navegación).

En cuanto a distribución de elementos y organización de los mismos en una cantidad de niveles razonable, es algo que no cumplen todas las RSV completamente, pero la que más se acerca es Metacafe. Esta RSV ofrece sólo 3 niveles de navegación, y posee una anchura máxima sólo de 17 que es bastante menor a la ofrecida por las otras RSV. Todas las RSV poseen una estructura similar ya que son sitios de un mismo dominio, pero la manera en la que organizan los elementos de navegación de esa estructura va a generar opciones de navegación y va a definir la cantidad de elementos en cada nivel.

Con respecto al análisis de la navegabilidad a partir de las diez guías seleccionadas, ninguna de las RSV logró cumplir con todas, lo que indica que cada RSV tiene diferentes aspectos a mejorar. Las RSV que cumplen entre el $40 \%$ y $60 \%$ de las guías. Youtube y Metacafe logran un mejor cumplimiento, y a la vez ambas cumplen con cuatro de las cinco guías con el NRI (Nivel Relativo de Importancia) más alto. Flooxer es la RSV que cumple con la menor cantidad de guías. También se puede destacar que de las tres guías con mayor NFE (Nivel de Fuerza de Evidencia), dos de ellas son cumplidas por Metacafe y Vimeo. Se puede afirmar que Metacafe cumple en gran medida con las guías con mayores valores de los indicadores NRI y NFI.

Los aspectos que se cubren en mayor cantidad por las RSV son: no dirigir a los usuarios a páginas que no tiene opciones de navegación (G01); diferenciar claramente los elementos de navegación entre sí (G02); y el uso de menús secuenciales y simultáneos de manera adecuada (G09). Las guías que se cubren en un 50\% son: proporcionar indicadores para que los usuarios sepan dónde están dentro del sitio (G04); colocar los menús de navegación primarios en el panel izquierdo y los menús secundarios juntos (G05); y proporcionar glosses para ayudar a los usuarios a seleccionar los enlaces correctos (G11). Los aspectos que le faltan cubrir a las RSV en términos de navegación en mayor medida son los expuestos por las siguientes guías: proporcionar una "lista de contenidos" con enlaces que lleven a los usuarios al contenido correspondiente en páginas largas (G03), esto no se cumple debido a las largas páginas de videos que presentan casi todas las RSV; no es necesario que los usuarios se desplacen en las páginas puramente de navegación (G08); utilizar sitemaps (G10), sólo la RSV Vimeo lo presenta; y proporcionar breadcrumb (G12), sólo la RSV Break lo proporciona.

Luego de todo el análisis se puede afirmar que la RSV que se encuentra mejor posicionada con relación a las otras RSV, en términos de navegabilidad es Metacafe. Esta RSV ha sobresalido en el análisis de su estructura con los sitemaps, y también en sus características de navegabilidad según la guía que fue aplicada.

Desde luego todo el análisis realizado en las RSV puede replicarse y aplicarse a otro tipo de sitios web. Así se puede obtener información similar que destaque debilidades y fortalezas de otro de tipo de sitio web. 


\section{REFERENCIAS}

BADRE, A.N. (2002). Shaping Web Usability: Interaction Design in Context. Boston, MA: Addison Wesley Professional. https://doi.org/10.1145/763913.763910

FLEMING J. (1998). Web navigation: designing the user experience. O'Reilly

GARENNE, B. (2018). Top 50 Sitemap Generator Tools for Creating Visual Sitemaps. 25/01/2018. Disponible en: https://dynomapper.com/blog/16-sitemap-generator/164top-50-sitemap-generators-for-creating-sitemaps (Último acceso: 07/12/2018)

HERRERA, R. y CODINA L. (2015), Redes Sociales Visuales: Caracterización, Componentes y posibilidades para el SEO de Sitios Intensivos en Contenidos. CDM (Cuaderno de Documentación Multimedia) Vol. 26 - ISNN: 1575-9733. https://doi.org/10.5209/rev_CDMU.2015.v26.50627

HOFMEISTER C. y HAN M. (2006). Modeling and Verification of Adaptive Navigation in Web Applications. Proceeding ICWE '06 Proceedings of the 6th international conference on Web engineering, (p.329-336)

HORNOF, A.J. y HALVERSON, T. (2003). Cognitive strategies and eye movements for searching hierarchical computer displays. CHI Conference Proceedings, 249-256. https://doi.org/10.1145/642611.642656

HULL, S.S. (2004), Influence of training and exposure on the usage of breadcrumb navigation, Usability News, 6.1. Disponible en: http://psychology.wichita.edu/surl/usabilitynews/61/breadcrumb.htm (Último acceso: 07/12/2018)

KINGSBURG, J.R. y ANDRE, A.D. (2004). A comparison of three-level web menus: Navigation structures. Proceedings of the Human Factors and Ergonomics Society 48th Annual Meeting. https://doi.org/10.1177/154193120404801309

LEAVITT M. O. y SHNEIDERMAN, B. (2004). Research-Based Web Design \& Usability Guidelines. Washington, U.S.: U.S. Government Printing Office. ISBN 0-16-076270-7

MONTERO, Y. H y ORTEGA SANTAMARÍA, S. (2009). Informe APEI sobre usabilidad. Director editorial: Raquel Lavandera Fernández.

MORROGH, E. (2002). Information architecture: an emerging 21st century profession. Upper Saddle River, NJ: Prentice Hall.

NIELSEN, J. (1990), 'The Art of Navigating Through Hypertext', Communications of the ACM, vol. 33, no. 3, (p. 296-310). https://doi.org/10.1145/77481.77483

NIELSEN J. (1999). Designing Web Usability: the practice of semplicity. New Riders Publishing

NIELSEN, J. (2000). Usabilidad. Diseño de sitios Web. Madrid, Ed. Pearson Educación S.A.

NIELSEN, J. y TAHIR, M. (2002). Homepage Usability: 50 Sites Deconstructed. Indianapolis, IN: New Riders Publishing.

NIELSEN J. (2008). Site Map Usability. . Disponible en: https://www.nngroup.com/articles/site-map-usability/ (Último acceso: 07/12/2018)

NIELSEN, J. (2011). Top ten mistakes in Web design. Disponible en: http://www.useit.com/alertbox/9605.html (Último acceso: 07/12/2018)

NORMAN, K.L. (1991), the Psychology of Menu Selection, Ablex Pub, N.J

PILGRIM, C. J. (2007). Trends in Sitemap Designs - A Taxonomy and Survey. Usability Innovation Group Faculty of Information and Communication Technologies, Swinburne University of Technology.

SITEMAPS.ORG (2008). ¿En qué consisten los Sitemaps? Disponible en: https://www.sitemaps.org/es/index.html (Último acceso: 07/12/2018) 


\begin{abstract}
ANEXO
Consultas ejecutadas:

a) Nombre del sitio = \{Youtube; Vimeo; Dailymotion; Metacafe; Break; Flooxer $\}$

Primer nivel: En este nivel hay nodos del tipo Categoria.

count $(/ /$ sitemap[@ nombre="Nombre del sitio"]/*)

Segundo nivel: En este nivel hay nodos del tipo Categoria y Sitio.

count(//sitemap[@ nombre="Nombre del sitio"]/categoria/*)

Tercer nivel: En este nivel hay nodos del tipo Categoria y Sitio.

count(//sitemap[@nombre="Nombre del sitio"]/categoria/categoria/*)

Cuarto nivel: En este nivel hay nodos del tipo Categoria y Sitio.

count(//sitemap[@ nombre="Nombre del sitio"]/categoria/categoria/categoria/*)

Quinto nivel: En este nivel hay nodos del tipo Categoria y Sitio.

count(//sitemap[@ nombre="Nombre del sitio"]/categoria/categoria/categoria/categoria/*)

b) Nombre del sitio = \{Youtube; Vimeo; Dailymotion; Metacafe; Break; Flooxer $\}$

Nivel 1:

for \$x in //sitemap[@ nombre="Nombre del sitio"]

return $\$ x / \operatorname{count}(*)$
\end{abstract}

Nivel 2:

for $\$ \mathrm{x}$ in //sitemap[@ nombre="Nombre del sitio"]/categoria

return $\$ \mathrm{x} / \operatorname{count}(*)$

Nivel 3:

for \$x in //sitemap[@ nombre="Nombre del sitio"]/categoria/categoria

return $\$ x / \operatorname{count}(*)$

Nivel 4:

for $\$ \mathrm{x}$ in //sitemap[ @ nombre="Nombre del sitio"]/categoria/categoria/categoria return $\$ x / \operatorname{count}(*)$

Nivel 5:

for \$x in //sitemap[@nombre="Nombre del sitio"]/categoria/categoria/categoria/categoria return $\$ x / \operatorname{count}(*)$ 